Originalveröffentlichung in: Christiane Zivie-Coche, Ivan Guermeur (Hg.), „Parcourir l'éternité“. Hommages à Jean Yoyotte, Turnhout 2012, S. 609-624

\title{
LIBYER UND ÄGYPTER IN DER LIBYERZEIT
}

\author{
Karl JANSEN-WinKELN*
}

1. Die Dritte Zwischenzeit, besonders die 22. Dynastie, wird in der Literatur auch gerne als „Libyerzeit“ bezeichnet. Aber was bedeutet das eigentlich? Auf den ersten Blick ist jedenfalls nicht viel Libysches oder Fremdes zu entdecken, und auch in den Darstellungen dieser Zeit spielt spezifisch Libysches kaum eine Rolle. Zwar gibt es Herrscher von offensichtlich libyscher Herkunft und auch nicht wenige andere Personen mit libyschen Namen ${ }^{1}$ sowie - sehr selten - einige libysche Titel, aber davon abgesehen finden wir kaum etwas, das als spezifisch libysch zu identifizieren wäre.

Das gilt ohne Einschränkung für Sprache und Texte: Alles Geschriebene ist ägyptisch geschrieben, die libysche Sprache ist nicht verschriftet. Wie wissen nicht einmal, ob es sich um eine Sprache oder um mehrere Sprachen oder Dialekte handelte. Aber es gibt auch keinen erkennbaren sprachlichen Einfluss auf das Ägyptische, so gut wie keine Fremd- oder Lehnwörter aus dieser Zeit, die man als Libysch identifizieren könnte ${ }^{2}$. Ähnlich ist es mit der Religion: Die in Ägypten ansässigen Libyern haben die ägyptische Religion übernommen, es sind keine exklusiv libyschen Götter bekannt noch sonst irgendwelche Merkmale, die man als libysch bestimmen könnte. Noch viel bemerkenswerter ist aber, dass es auch keinerlei archäologische Relikte gibt, die man den Libyern zuweisen könnte. Alle Objekte dieser Zeit, welcher Art auch immer, aus Tempeln und Bestattungen, die Keramik ${ }^{3}$ und andere Gegenstände des täglichen Lebens, sind ägyptischen Ursprungs: ihre Entwicklung kann zurückverfolgt werden, ohne dass größere Brüche festzustellen wären. Es ergibt sich ein erstaunlicher

\footnotetext{
* Außerplanmäßiger Professor an der Freien Universität Berlin.

1. Fr. Colın, Les Libyens en Égypte (Xve siècle A. C. $-I^{e}$ siècle P. C.). Onomastique et Histoire, 2006, vorläufig unter http:/tel.archives-ouvertes.fr.tel-00120038 einsehbar.

2. Angesichts der Tatsache, dass ein ganz erheblicher Teil des koptischen Wortschatzes bis heute ohne bekannte Etymologie ist, besteht allerdings die Möglichkeit, dass hier noch vieles unbekannt ist.

3. D. A. Aston, Egyptian Pottery of the Late New Kingdom and Third Intermediate Period (SAGA 13), Heidelberg, 1996, S. 59-89. Man beachte, dass Aston unter "foreign pottery“ (S. 83-86) nur „Greek pottery“, Cypriote Pottery“ und „Levantine Pottery“ "aufführt; libysche Keramik wird nicht erwähnt.
} 
Befund: Die jahrhundertelang dauernde Libyerzeit ist tatsächlich als solche archäologisch nicht nachweisbar ${ }^{4}$.

Paradoxerweise scheinen die Libyer in der Libyerzeit keine große Rolle zu spielen. Mehr noch: Die Libyer sind in der Zeit vor ihrer Herrschaft in den ägyptischen Quellen wesentlich prominenter. Das zeigt sich sehr deutlich in den Darstellungen, in denen die Rolle Libyens und der Libyer in der ägyptischen Geschichte insgesamt behandelt wird: W. Hölscher untersuchte in seiner Monographie ${ }^{5}$ Stämme, Trachten, Wohngebiete und Geschichte der Libyer bis zum Ende des Neuen Reiches auf den Seiten 1-66. Die Zeit danach, als die Libyer für Jahrhunderte Ägypten beherrschen, wird auf den Seiten 67-68 abgehandelt. Im Lexikon der Agyptologie hat J. Osing den Libyern einen ausführlichen Artikel von 17 Spalten gewidmet ${ }^{6}$. Die Zeit nach dem Neuen Reich wird darin allerdings nur in 4 Sätzen erwähnt. Eine umfangreiche Abhandlung von D. O'Connor über die Art der libyschen Gesellschaft ${ }^{7}$ beschränkt sich sogar ganz auf die Ramessidenzeit. Die Zeit nach dem Neuen Reich, als die Libyer die Herren des Landes waren, spielt in all diesen Darlegungen so gut wie keine Rolle.

Das ist natürlich nicht ohne Grund so: Nur aus dem Neuen Reich haben wir etwas mehr Informationen über Aussehen, Tracht und Waffen der Libyer, über die Beschneidung bei verschiedenen Stämmen, über die von ihnen bei Kriegszügen oder Wanderungen mitgeführten Objekte etc., und zwar hauptsächlich aus Texten und Bildern, in denen sie als Feinde der Ägypter erscheinen, wie in den Wandreliefs von Medinet Habu ${ }^{8}$.

2. Aus dem weitgehenden Fehlen libyscher Spuren in der Überlieferung hat man früher geschlossen, die Libyer seien in der Zeit, als sie Ägypten beherrschten, bereits weitgehend akkulturiert (,,ägyptisiert") gewesen, und die Ägypter hätten diese Könige und Lokalfürsten folglich nicht als Fremde angesehen. Daher habe sich auch, abgesehen von der Herkunft dieser Herrscher, nicht allzu viel im Land verändert, dass auf libyschen Einfluß zurückzuführen sei. In jüngerer Zeit ist aber mehrfach gezeigt worden, dass die Argumente für eine Akkulturierung der Libyer nicht überzeugend sind und die libyschen Herrscher im Gegenteil ihren eigenen Vorstellungen und Traditionen folgten ${ }^{9}$.

\footnotetext{
4. Man muss allerdings im Auge behalten, dass einige Bereiche (Kleidung, Gebräuche) mithilfe unserer Quellen nicht zu erfassen sind.
}

5.W. HölsCher, Libyer und Ägypter. Beiträge zur Ethnologie und Geschichte libyscher Völkerschaften nach den altägyptischen Quellen (ÄF 4), Glückstadt, 1937.

6. J. OsING, in: LÄ III, Sp. 1015-1033, s.v. „Libyen, Libyer“.

7. D. O'Connor, „The nature of Tjemhu (Libyan) Society“, in: A. Leahy (ed.), Libya and Egypt c1300-750 BC, London, 1990, S. 29-113.

8. Nur ganz selten werden sie im Neuen Reich nicht als Feinde gezeigt, wie in der berühmten Szene des Pfortenbuchs, s. E. Hornung, Tal der Könige, Zürich - München, 1982, S. 147, Abb. 120.

9. A. LeAHY, „The Libyan Period in Egypt: An Essay in Interpretation“, LibStud 16 (1985), S. 51-65; K. JANSEN-WINKELN, „Die Fremdherrschaften in Ägypten im 1. Jahrtausend v. Chr.“, Or 69 (2000), S. 3-13; R. K. RitneR, „Fragmentation and Re-integration in the Third Intermediate Period“", in: G. P. F. Broekman - R. J. Demarée - O. E. Kaper (ed.), The Libyan 
Als wichtigste Argumente gegen eine (weitgehende) Akkulturierung der Libyer lassen sich anführen:

- Die Existenz und lange Dauer libyscher „Fürstentümer“ in Unterägypten, deren Oberhäupter sich selbst als $w r$ ` (,Großfürsten“ bzw. „Häuptlinge“) der $M(s ̌ w s ̌)$ oder der $R b w$ bezeichnen. Sie machen keinerlei Versuch, ihre Identität als Stammeshäuptling zu verleugnen, sondern lassen sich ganz im Gegenteil auch so darstellen, mit der Häuptlingsfeder ${ }^{10}$. R. K. Ritner hat überzeugend gezeigt, dass sich auch die zentrifugalen Kräfte, die diesem Herrschaftssystem innewohnen, am besten aufgrund libyscher Traditionen erklären lassen ${ }^{11}$.

- Königliche Prinzen werden als libysche Häuptlinge eingesetzt: Auf einem Stelophor Osorkons II. ${ }^{12}$ bittet der König Amun von Tanis, seine Nachkommen zu großen Herrschern von Ägypten zu machen, Prinzen $\left(j r j-p^{t} t\right)$, Hohenpriestern des Amun, Großfürsten der Ma ( $w r$ ` $\left.n M\right)$, Großen der Ausländer (wrw nhjstjw) und zu Propheten des Herischef. Die gleiche Bitte findet sich fast im Wortlaut auch auf einem Block Osorkons I. aus Bubastis ${ }^{13}$. Interessanterweise werden in beiden Texten nicht nur die Großfürsten der Meschwesch erwähnt, sondern auch Großfürsten der „Ausländer“" (hjstjw). Diese gleiche Bezeichnung kommt auch sonst einmal in der Königsfamilie vor: eine Frau Schoschenks I.(?) ist die Tochter eines Großfürsten der Ausländer ${ }^{14}$.

- Der Hohepriester von Memphis Pediese wird auf seinen Serapeumstelen aus dem Jahr 2 des Pami als Hoherpriester und als Häuptling der Meschwesch bezeichnet ${ }^{15}$, auf einer weiteren aus dem Jahr 28 Schoschenks III. sogar nur als Häuptling der Meschwesch ${ }^{16}$, und er wird auch so dargestellt. Diese Stelen wurden anlässlich des Begräbnisses des Apisstiers errichtet, eine der wichtigsten und traditionsreichsten Angelegenheiten des ägyptischen Kultus. Dennoch wird hier sichtbar die libysche Identität des obersten Wächters dieses

Period in Egypt, Historical and Cultural Studies into the $21^{\text {st }}-24^{\text {th }}$ Dynasties: Proceedings of a Conference at Leiden University, 25-27 October 2007 (EgUit 23), Löwen, 2009, S. 327-340.

10. A. Leahy, „The Libyan Period in Egypt“, S. 57; K. JANSEN-WinKeln, „Die Fremdherrschaften“, S. 8; R. K. RITNER, „Fragmentation and Re-integration in the Third Intermediate Period“, S. 336337.

11. Ibid., S. 327-340. Das bedeutet natürlich nicht, daß die Libyer bewußt auf eine Fragmentierung des Landes hingearbeitet hätten, wie das die Kritik von M. HüNEBURG, ,,Soziologische Überlegungen zum Partikularisierungsprozeß Ägyptens nach dem Ende des Neuen Reiches und während der Dritten Zwischenzeit“, in R. Gundlach - U. Rößler-Köhler, Das Königtum der Ramessidenzeit, Akten des 3. Symposiums zur ägyptischen Königsideologie in Bonn 7. - 9. 6. 2001 (ÄAT 36,3), Wiesbaden, 2003, S. 57-75 unterstellt. Seine alternative Erklärung betrifft zudem nur allgemeine Herrschaftstechniken, nicht die konkrete historische Situation.

12. Kairo CG 1040, s. H. JACQUET-GoRDon, „The Inscriptions of the Philadelphia - Cairo Statue of Osorkon II“", JEA 46 (1960), S. 12-23; pl. VII-VIII; K. JANSEN-WINKELN, Inschriften der Spätzeit, Teil II: Die 22.-24. Dynastie, Wiesbaden, 2007, S. 108-109.

13. E. LANGE, „Legitimation und Herrschaft in der Libyerzeit“, ZÄS 135 (2008), S. 131-141; Taf. XXXIV-XXXIX.

14. K. JANSEN-WINKELN, Inschriften der Spätzeit II, S. 84-85 (Nr.1-3).

15. M. Malinine - G. Posener - J. Vercoutter, Catalogue des stèles du Sérapéum de Memphis, Paris, 1968, S. 21-23; pl. 8 (Nr. 22-23).

16. Ibid., S. 19-20; pl. 7 (Nr. 21). 
Kults demonstriert ${ }^{17}$. Der Vater und Amtsvorgänger des Pediese namens Takeloth (B) führt gleichfalls lieber den Häuptlingstitel eines $w r$ \ $n$ Mšwš statt des Hohenpriestertitels, auch in seiner Grabausstattung ${ }^{18}$. Aufschlussreich sind auch die genealogischen Beziehungen: der Häuptling Takeloth (B) ist der Sohn des Hohenpriesters von Memphis und Kronprinzen Schoschenk D und damit der Enkel Osorkons II. Die Mitglieder der Königsfamilie, die höchste Priesterämter in Memphis einnehmen, sehen sich also in der 6. und 7. Generation nach Beginn der 22. Dynastie noch als libysche Stammesführer.

- Der Priester Pasenhor, der die bekannte Stele aufstellen lie $\beta^{19}$, betont nicht nur seine Abstammung aus der Königsfamilie, sondern auch deren und damit seine eigene Abstammung von einem Libyer! Das war offenbar etwas, worauf er stolz war.

Bei näherem Hinsehen hat man keineswegs den Eindruck, als hätten sich die Herrscher der 22. Dynastie als Ägypter gefühlt, sie sahen sich selbst zweifellos als Libyer.

3. Wenn aber Herrscher und Teile der Oberschicht Fremde waren und sich selbst auch so sahen, sollte man große Veränderungen im Land erwarten, neue Strukturen, die den Traditionen und Interessen der neuen Herren angemessen waren. Gibt es also tatsächlich in der Libyerzeit wichtige strukturelle Veränderungen? Solche Veränderungen aufzuspüren und zu erkennen ist ganz allgemein für die Rekonstruktion der ägyptischen Geschichte eines der wesentlichsten Mittel, denn in Ägypten gab es ja vor Manetho keine Geschichtsschreibung, die größere Zeiträume umfasst und Zusammenhänge deutlich macht. Und inschriftliche Äußerungen dürfen wir zu vielen, ja den meisten Vorgängen gar nicht erwarten: Königsinschriften oder Biographien berichten nur von erfolgreichen Taten, Niederlagen, Invasionen und alle Arten von Misserfolgen bleiben unerwähnt. Oft bleibt nur die Möglichkeit, aus strukturellen Veränderungen Schlüsse zu ziehen, um wenigstens die grobe Richtung des Ablaufs der Dinge zu erahnen.

Die auffälligste strukturelle Veränderung gegenüber dem Zustand im Neuen Reich ist die getrennte Verwaltung und Entwicklung in Ober- und Unterägypten ${ }^{20}$. Für Oberägypten gibt es einen Regenten, dem das Land von Elephantine bis in die Gegend südlich des Fayum (bei El-Hibe) untersteht. Er ist Oberster Militärführer und zugleich Hoherpriester des Amun von Theben. Aufgrund der einseitigen Art unserer Quellen, die ja fast ausschließlich aus Tempeln und Gräbern stammen, wird diese Person häufig auch einfach als „Hoherpriester des Amun“ bezeichnet, und auch in der ägyptologischen Literatur hat man sich davon vielfach täuschen lassen und hält ihn in erster Linie für einen „Priester“. Das ist mit Sicherheit falsch: Sein wichtigeres, eigentliches Amt ist sicher das des Oberbefehlshabers, dem dann zugleich,

17. R. K. Ritner, „Fragmentation and Re-integration in the Third Intermediate Period“, S. 336; K. JANSEN-WINKELN, „Die Fremdherrschaften“, S. 8.

18. Tanis. L'or des pharaons, Ausstellungskatalog Paris, 1987, S. 150-151 (34).

19. M. Malinine - G. Posener - J. Vercoutter, Catalogue des stèles du Sérapéum, S. 30-31; pl. 10 ; K. A. KItchen, The Third Intermediate Period in Egypt, Warminster, 1972, S. 488 (table 19).

20. Vgl. K. JANSEN-WINKELn, „Der thebanische 'Gottesstaat'“, Or 70 (2001), S. 160. 
als wirtschaftliche Grundlage seiner Macht, die Tempelländereien des Amun unterstehen ${ }^{21}$.

In Unterägypten ist die Situation vollkommen anders: ab dem 9. Jahrhundert wird deutlich, dass es zum einen königliche Residenzstädte gibt (wie Tanis, Bubastis und Leontopolis), zum anderen zahlreiche Territorien, die von einem Großfürsten der Meschwesch (im zentralen und östlichen Delta) oder der Libu (im westlichen Delta) mehr oder weniger selbständig regiert werden ${ }^{22}$. Diese „Großfürsten“ ( $w r$ (3) sind also Stammesführer, ihren ägyptischen Titeln nach aber auch wiederum oberste Militärbefehlshaber ihres Gebiets und Hohepriester des wichtigsten Kultes darin. In Oberägypten sind dagegen keine libyschen Häuptlinge als Lokalherrscher bezeugt.

Ober- und Unterägypten haben also ein vollkommen verschiedenes Herrschaftssystem. Eine derartige Teilung Ägyptens hat es in früheren Epochen nur dann gegeben, wenn Unterägypten unter fremder Herrschaft stand, wie unter den Hyksos (und vermutlich auch in der 1. Zwischenzeit). Auch im Selbstverständnis der Herrscher hat sich einiges verändert: Auf der Pianchistele finden wir bekanntlich nicht weniger als 5 Könige sowie 5 Lokalfürsten, die alle zugleich über bestimmte Gebiete Ägyptens herrschen, in den Assurbanipalannalen werden sogar 16 Herrscher für Unterägypten und 4 für Oberägypten aufgezählt. Der König (njswt) ist also nur noch eine Art besonders hervorgehobener Fürst, und diese Erscheinung findet sich auch schon deutlich früher in der 3 . Zwischenzeit ${ }^{23}$. Mit der älteren ägyptischen Tradition ist das unvereinbar.

Die Verwaltung des Landes ist auch auf der mittleren und unteren Ebene ganz anders geregelt als noch im Neuen Reich (und allen vorherigen Epochen): Die meisten Titel der Landes- und Lokalverwaltung des Neuen Reiches sind nicht mehr bezeugt ${ }^{24}$. Dagegen sind nahezu alle Mitglieder der ägyptischen Oberschicht, die uns Schriftliches hinterlassen haben, Priester, entweder im Hauptberuf oder neben ihren Schreiber- und Verwaltungstätigkeiten.

Eine radikale Veränderung lässt sich auch bei den Bestattungssitten feststellen: In der Dritten Zwischenzeit überwiegen bei weitem die Sammelbestattungen in alten, wiederbenutzen Gräbern oder einfachen, undekorierten Grüften ${ }^{25}$. Neue dekorierte Gräber für Privatpersonen werden nach dem neuen Reich so gut wie gar nicht mehr angelegt, erst in der

21. Sein Amt als Priester hat er allenfalls an besonders hohen Festen wahrgenommen; zumindest in einem Fall erfahren wir das auch expressis verbis: in den „Osorkonannalen“ sagt der Hohenpriester Osorkon B, dass er (nur) dreimal im Jahr nach Theben kam, s. THE EPIGRAPHIC SURVEY, Reliefs and Inscriptions at Karnak III, The Bubastite Portal (OIP 74), Chicago, 1954, pl. 21, Z. 6; R. A. Caminos, The Chronicle of Prince Osorkon (AnOr 37), Rom, 1958, S. 78.

22. J. YоYотте, „Les principautés du Delta au temps de l'anarchie libyenne (études d'histoire politique)“", in Mélanges Maspero I/4 (MIFAO 66/4), Kairo, 1961, S. 121-181; F. GomaÀ, Die libyschen Fürstentümer des Deltas vom Tod Osorkons II. bis zur Wiedervereinigung Ägyptens durch Psametik I. (TAVO B/6), Tübingen, 1974.

23. Schon in der 21. Dynastie führen zeitweise mehrere Herrscher den Königstitel.

24. K. JANSEN-Winkeln, „Die Fremdherrschaften in Ägypten“, S. 11; ID., „Gab es in der altägyptischen Geschichte eine feudalistische Epoche“, WdO 30 (1999), S. 16.

25. S. dazu die umfassende Zusammenstellung des Materials durch D. A. Aston, Burial Assemblages of Dynasty 21-25. Chronology - Typology - Developments (DÖAWW 56), Wien, 2009. 
späteren Dritten Zwischenzeit gibt es wieder ganz vereinzelt ein paar kleine Grabkapellen. Ab der späteren 25. Dynastie werden dann in Theben wieder sehr große dekorierte Grabanlagen gebaut, aber nur für höchste Würdenträger. Die Bestattung in älteren wiederbenutzten Gräbern bleibt die ganze Spätzeit hindurch der Regelfall. Das Grab wird nie mehr in dem Maße Prestigeobjekt wie in den früheren Epochen der ägyptischen Geschichte. Diese radikale Veränderung der Bestattungssitten findet sich ebenso bei den Königsgräbern: Die sind jetzt - verglichen mit allen früheren Epochen - geradezu winzig klein und aus wiederverwendeten Bauteilen errichtet (vermutlich erst unmittelbar vor der Bestattung). Auch königliche Totentempel gibt es nicht mehr. Bei den Königsgräbern können wir also einen glatten Bruch mit der gesamten Tradition königlicher Bestattungen seit dem frühen Alten Reich konstatieren.

Man hat vielfach versucht, diese durchgreifenden Veränderungen der Bestattungssitten mit den Grabräubereien am Ende des Neuen Reiches zu erklären, aber das ist nicht überzeugend: Zum einen hat es Grabräubereien $\mathrm{zu}$ allen Zeiten gegeben, zweitens erklären sie nicht, warum man keine dekorierten Gräber mehr angelegt hat, denn wenn eine Bestattung beraubt wird, erhält gerade die Dekoration die Erinnerung an den Toten aufrecht. Und das Grab als gesellschaftliches Prestigeobjekt ist ja auch und vor allem zu Lebzeiten des Besitzers von Bedeutung. Die Veränderungen deuten also in erster Linie auf einen Wandel in den Wertvorstellungen der Oberschicht.

Einen tiefgreifenden Wandel bezeugt auch die Namensgebung: In der Dritten Zwischenzeit treffen wir nicht nur erstmalig libysche Namen in größerem Umfang an, auch das Bild der ägyptischen Personennamen verändert sich sehr stark und nachhaltig: besonders häufig sind jetzt bestimmte Namenstypen, die alle im Neuen Reich noch nicht vorkommen, z.B.

$P_{j}$-dj-Jmn / T3-dj-Jmn, Jmn-jrj-dj-sw/sj, Pj-šrj-n-Jmn / T3-šrjt-n-Jmn,

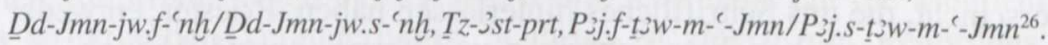

Ein weiterer Punkt, an dem sich eine Veränderung im Selbstverständnis der Oberschicht zeigt, sind die langen Genealogien auf Privatdenkmälern, die im Neuen Reich noch so gut wie gar nicht vorkommen: die Filiationsangaben beschränken sich dort weitestgehend auf die Eltern. In der 3. Zwischenzeit dagegen finden sich häufig Stammbäume, die über drei, fünf, zehn oder noch mehr Generationen zurückreichen. A. Leahy hat diese ausführlichen Stammbäume auf libyschen Einfluss zurückgeführt, da die mündliche Tradierung der Abstammung typisch für nichtliterate Stammesgesellschaften $\mathrm{sei}^{27}$. R. K. Ritner hat das noch weiter vertieft ${ }^{28}$. Ihm zufolge ist die zentrifugale Entwicklung im Ägypten der späteren 3. Zwischenzeit (neben

26. Vgl. $P N$ II, S. 243-244. Auch die Bildung $P j-n-J m n / T 3-n(t)-J m n$ ist sehr häufig und kommt besonders häufig nach dem Neuen Reich vor. Insgesamt ist die Änderung der Namen ist so durchgreifend, dass ihre Form oft für eine grobe Datierung ausreicht: Findet man auf einem Objekt einen solchen Namen, stammt es aus der Zeit nach dem Neuen Reich, und finden sich umgekehrt mehrere Personennamen, von denen keiner zu diesen eben erwähnten Typen gehört, ist das Objekt sehr wahrscheinlich älter als die Dritte Zwischenzeit.

27. A. Leahy, „The Libyan Period in Egypt“, S. 55.

28. R. K. RITNER, „Fragmentation and Re-integration in the Third Intermediate Period“, S. 327-340. 
anderen Erscheinungen) am besten dadurch zu erklären, dass die libyschen Herrscher nach dem für viele Stammesgesellschaften typischen Modell der "segmentary lineages“ organisiert waren: Brüder und Vettern einer Abstammungslinie stehen gegen äußere Feinde zusammen, sind aber intern in ständiger Konkurrenz um die Vorherrschaft. In jedem Fall aber ist in solchen Gesellschaften die Abstammung von überragender Bedeutung.

Das Ägypten der Libyerzeit ist also in vielerlei Hinsicht ganz anders strukturiert ist als das Ägypten des Neuen Reiches.

4. Bisher war von der Libyerzeit oder der 3. Zwischenzeit die Rede, als sei damit dieselbe Epoche gemeint, aber gerade das ist eines der wichtigsten Probleme: Die 3. Zwischenzeit beginnt nach allgemeiner Ansicht mit der 21. Dynastie, die Libyerzeit aber, zumindest nach traditionellem Verständnis, erst mit der 22. Auch die Frage, wie die Libyer überhaupt an die Macht gelangten, ist keineswegs geklärt. Wann und wie ist also die Libyerherrschaft entstanden? Die Ausgangslage für diese Frage ist zunächst einmal die gleiche wie für viele, ja die meisten historischen Probleme dieser Zeit: Es gibt keine Quellen, die uns ohne weiteres sagen, wann und wie die Übernahme der Macht durch libysche Herrscher vor sich gegangen ist. Wir sind wiederum auf indirekte Schlüsse angewiesen.

Früher war es unstrittig, dass die Herrschaft der Libyer mit der 22. Dynastie begonnen hat: Die Pasenhorstele führt die vier ersten Könige dieser Dynastie in Vater-Sohn-Folge auf, beginnend mit Schoschenk I. Dessen Vater war aber nur ein „Großfürst“" ( $w r$ (s), ebenso wohl seine älteren Vorfahren. Daraus, wie aus der Tatsache, dass die Könige der 21. Dynastie noch ägyptische Namen tragen, hatte man geschlossen, dass Schoschenk I. der erste libysche Pharao gewesen ist. Man hat sich daher die Machtübernahme durch die Libyer gern folgendermaßen vorgestellt: Nach den Siegen Ramses' III. sei die unmittelbare militärische Bedrohung durch die Libyer beendet gewesen, aber es habe in der 2. Hälfte der 20. und während der 21. Dynastie eine ständige Zuwanderung gegeben. Zudem hatte Ramses III. ja schon Libyer und andere Kriegsgefangene in Festungen ansiedeln lassen, die Libyer haben schon unter den Ramessiden in größerer Zahl als Söldner gedient. Sie seien also im Verlauf der 20. und 21. Dynastie in Ägypten zahlreicher und einflussreicher geworden, hätten sich zugleich weitgehend akkulturiert und schließlich mit Schoschenk I. auch formal die Macht übernommen, dies alles überwiegend auf friedlichem Weg.

Inzwischen haben sich aber neue Fakten und Aspekte ergeben, die diese Rekonstruktion weniger überzeugend erscheinen lassen:

J. Yoyotte hatte schon vor mehr als 30 Jahren gezeigt, dass Schoschenk I. nicht der erste libysche Pharao war: der drittletzte Pharao der 21. Dynastie war ein Onkel Schoschenks I. namens Osorkon (Osochor bei Manetho) ${ }^{29}$. Und in der Herrscherfamilie der 21. Dynastie erscheinen auch sonst schon

29. J. Yочотте, ,'Osorkon fils de Mehytouskhé’, un pharaon oublié?“, BSFE 77-78 (1976-1977), S. 39-54. Dieser brillante Aufsatz hat weitreichende Folgen für das Verständnis der gesamten Epoche gehabt. Und er ist ein gutes Beispiel dafür, wie J. Yoyotte durch scharfsinniges Erfassen und Einordnen scheinbarer Kleinigkeiten, verbunden mit einer umfassenden Kenntnis des Materials und der Literatur, immer wieder neue weitreichende Erkenntnisse und oft geradezu revolutionäre Einsichten vorgelegt hat. 
früh libysche Namen, wie bei dem General und Hohenpriester Masaharta, einem Sohn Pajnedjems I., und einigen Söhnen des $\mathrm{Herihor}^{30}$. Wesentlich wichtiger aber ist etwas anderes: Die Herrschaft der Libyer hat zu tiefgreifenden Veränderungen in Ägypten geführt, und zwar in ganz unterschiedlichen Bereichen (s.o., § 4). Alle diese Veränderungen setzen aber nicht erst mit der 22. Dynastie ein, sondern schon mit der 21.:

Die Teilung und unterschiedliche Verwaltung des Landes beginnt in der 21. Dynastie. Seit dieser Zeit wird Oberägypten von einem Militärbefehlshaber regiert, der zugleich Hoherpriester des Amun ist und in Theben und in El-Hibe residiert, genau wie in der 22. Dynastie.

Allerdings sind in der 21. Dynastie in Unterägypten die „Großfürsten“ der libyschen Stämme noch nicht bezeugt, erst in der späteren 22. Dynastie ab Schoschenk III. finden wir sie als Lokalherrscher, v.a. auf Schenkungsstelen, Statuen und Architekturfragmenten. Das dürfte einerseits durch die Quellenlage, andererseits durch bestimmte Entwicklungen bedingt sein:

Denkmäler der 21. Dynastie gibt es in Unterägypten fast nur in der Königsstadt Tanis und in Memphis, und Schenkungsstelen sind aus dieser Zeit überhaupt nicht bekannt. Auch aus der früheren 22. Dynastie sind aus Unterägypten außerhalb der „Königsstädte“ Tanis und Bubastis nur wenige Funde bekannt. Die wenigen Schenkungsstelen aus dieser Zeit zeigen zudem alle noch den König als Stifter (bzw. Mittler). Dass aber schon in der frühen 22. Dynastie die „Großfürsten der Meschwesch“ eine wichtige Rolle spielten, zeigen zwei Inschriften: Auf einem Block Osorkons I. aus Bubastis ${ }^{31}$ bittet der König die Bastet, seine Nachkommen einzusetzen als Könige, Hohepriester des Amun und des Herischef sowie als Großfürsten der Ma und der „Ausländer“ (hjstjw), und diese Passage kommt fast wortgleich auf einer Statue Osorkons II. in einem Gebet an Amun von Tanis vor ${ }^{32}$. Spätestens ${ }^{33}$ ab Osorkon I. stehen also libysche Großfürsten bzw. Häuptlinge als Teilherrscher Ägyptens neben den militärischen und ,geistlichen " Führern in Theben und Herakleopolis, die ihrerseits (im besser dokumentierten Oberägypten) schon in der 21. Dynastie belegt sind. Es ist daher sehr wahrscheinlich, dass wir "libysche Großfürsten“ nur aufgrund mangelnder Quellen im Unterägypten der 21. und frühen 22. Dynastie nicht nachweisen können ${ }^{34}$.

Auch die Erscheinung, dass die Zivilverwaltung verschwindet und bei den Ägyptern fast nur noch Priester- und Schreibertitel zu finden sind,

30. Fr. Colin, Les Libyens en Égypte I, S. 26; 95; 97.

31. E. LANGE, ,Legitimation und Herrschaft in der Libyerzeit“, S. 131-141.

32. H. JACQUET-GORDON, „The Inscriptions of the Philadelphia - Cairo Statue of Osorkon II“, S. 16-17; K. JANSEN-WINKELN, Inschriften der Spätzeit II, S. 108-109.

33. Aufgrund der Übereinstimmungen der Texte ist es sogar wahrscheinlich, dass es eine noch ältere Vorlage für diese Passage gegeben hat.

34. Vgl. in diesem Sinne auch A. LEAHY, ,The Libyan Period in Egypt“, S. 54. Da gerade diese Häuptlinge in der Kontinuität libyscher Traditionen stehen, sollten sie mindestens ebenso früh vorhanden gewesen sein wie die regierenden Hohenpriester und Armeeführer in Theben und Herakleopolis. Es ist nicht sehr verwunderlich, dass sie erst nach längerer Zeit (in ägyptischer Tradition) als Bauherren und Stifter von Statuen in Erscheinung getreten, später sogar (auf den Schenkungsstelen) als Stellvertreter des Königs. 
lässt sich schon deutlich in der 21. Dynastie feststellen ${ }^{35}$. Bemerkenswert in diesem Zusammenhang ist der Block Kairo JE 39410 ${ }^{36}$, auf dem die Personen und Institutionen aufgelistet sind, die verpflichtet sind, das tägliche Opfer für Herischef von Herakleopolis zu unterhalten. Es handelt sich um Militärpersonal, Priester und Handwerker sowie ganze Ortschaften. Ämter und Personen der Staats- oder Lokalverwaltung werden hingegen nicht genannt, es gibt sie offenbar nicht mehr. Und da das Objekt aus der Zeit Schoschenks I. stammt, muss dieses System schon zu Beginn der 22. Dynastie voll ausgebildet gewesen sein. Das spricht dafür, dass es schon längere Zeit vor Schoschenk I. eine Entwicklung in diese Richtung gegeben hat.

Die für die Libyerzeit typische Erscheinung, dass der König nur noch ein besonders hervorgehobener Fürst ist, lässt sich schon in der 1. Hälfte der 21. Dynastie beobachten: mehrere Herrscher Oberägyptens bezeichnen sich nicht nur als Militärbefehlshaber und Hohepriester, sondern daneben und zugleich auch als Könige. Und auch die Zusammenfassung von militärischer und geistlicher Macht in einer Person ist charakteristisch. Sie findet sich bei den libyschen „Großfürsten“" in Unterägypten ${ }^{37}$ ebenso wie bei den (schon in der 21. Dynastie bezeugten) oberägyptischen Militärführern und Hohenpriestern ${ }^{38}$.

Ebenfalls in den Beginn der 21. Dynastie zurück geht die radikale Änderung der Bestattungssitten, nämlich das systematische Usurpieren alter Gräber und die Anlage von Sammelgräbern statt des Baus neuer dekorierter Gräber. Auch die Aufgabe der thebanischen Königsnekropole und der gesamten älteren Tradition königlicher Bestattungen in großangelegten Gräbern mit jeweils eigenen Totentempeln endet mit Ramses XI., und die neue Tradition unscheinbarer Königsgräber im Tempelbereich beginnt mit der frühen 21. Dynastie. Die neue Nekropole in Tanis zeigt auch, wie eng verwandt die 21. und 22. Dynastie sind: die Könige beider Epochen sind in demselben Gräberkomplex beigesetzt worden, und die Gräber selbst sind in Machart (aus Spolien errichtet) und Ausstattung (mit usurpierten Sarkophagen) ganz gleichartig.

Die neuen Namenstypen, die so charakteristisch für die Zeit nach dem Neuen Reich sind, finden sich gleichfalls schon überaus zahlreich in der 21. Dynastie. Etwas anders sieht es mit den längeren Stammbäumen aus: sie sind in der Hauptsache auf Statuen und Steinstelen von Privatleuten zu finden, und die sind in der 21. Dynastie nicht bezeugt. Immerhin finden wir auf Totenpapyri

35. Vgl. etwa die Zusammenstellung thebanischer Titel bei A. NiwINSKI, $27^{s t}$ Dynasty Coffins from Thebes. Chronological and Typological Studies (Theben V), Mainz, 1988, S. 192-194.

36. P. Tresson, „L'inscription de Chechanq Ir, au Musée du Caire: un frappant exemple d'impôt progressif en matière religieuse", in Mélanges Maspero I/2 (MIFAO 66/2), Kairo, 1938, S. 817-840; K. JANSEN-WINKELN, Inschriften der Spätzeit II, S. 4-7; ID., „Die Libyer in Herakleopolis magna“, Or 75 (2006), S. 297-300; R. MEFFre, „Un nouveau nom d'Horus d'or de Sheshonq Ir sur le bloc Caire JE $39410 “$, BIFAO 110 (2010), S. 221-233.

37. J. Yoyotre, „Les principautés du Delta“, S. 139, § 21-22.

38. Gemeinsam ist den ober- wie den unterägyptischen Teilherrschern, dass sie 1. zur Herrscherfamilie gehören, 2. die Militärführer ihres Gebietes sind und 3. Oberste Priester des Hauptkultes, also geistliche Autorität und oberstes Kontrollorgan der Priester und der wirtschaftlichen Ressourcen der Tempel. 
dieser Zeit ein paar Fälle ${ }^{39}$, wo die Vorfahren über drei oder vier Generationen angeführt werden, was aus dem Neuen Reich noch nicht bekannt ist.

Sofern uns die wenigen Quellen überhaupt ein Urteil gestatten, setzen alle für die Libyerzeit charakteristischen Veränderungen schon mit der 21. Dynastie ein. Und auch diese Quellenlage selbst enthält einen wichtigen Hinweis: Gerade aus der 21. Dynastie ist besonders wenig überliefert, wir haben zum einen die Königsgräber in Tanis, zum anderen die thebanischen Sammelgräber - und sonst fast nichts. Auch die Kategorien von Denkmäler, die vom Neuen Reich an bis in die Spätzeit so reich bezeugt sind, wie Privatstatuen, Schenkungsstelen und Serapeumstelen, sind alle in der 21. Dynastie nicht belegt, hier haben wir eine große Lücke in der Dokumentation. Und das ist wiederum typisch für den Beginn von Fremdherrschaften, es findet sich beispielsweise ebenso in der 27. Dynastie ${ }^{40}$.

Für die Frage, wann die Herrschaft der Libyer entstanden ist, scheint alles auf den Beginn der 21. Dynastie hinzudeuten, dem Zeitpunkt, als die großen strukturellen Änderungen einsetzen.

5. In diesem Fall ist aber damit zugleich schon fast die Frage nach dem wie beantwortet: denn die Übernahme der Macht wäre dann recht plötzlich erfolgt, es wäre keine schleichende „Machtergreifung“ durch allmähliche Zuwanderung gewesen, wie man oft annimmt. Und das wiederum würde fast gewiss bedeuten: die Übernahme der Macht erfolgte gewaltsam, durch Eroberung. Der Einwand, dass wir für eine gewaltsame Machtübernahme der Libyer am Ende des Neuen Reiches keine Quellen haben, zählt nicht viel, denn solche Quellen sind a priori gar nicht zu erwarten. Die Könige der späteren 20. Dynastie hätten Niederlagen gegen libysche - oder andere Eroberer sicher nicht in Stein meißeln lassen.

Hingegen könnten sich indirekte Hinweise auf derartige Vorgänge finden: In thebanischen Papyri der späten 20. Dynastie werden öfter „Barbaren“ (hıstjw) oder konkret Libyer vom Stamme der Meschwesch und Libu in Theben erwähnt, meist als Grund für die Untätigkeit der Nekropolenarbeiter ${ }^{41}$. Diese Erwähnungen von Fremden in Theben sind früher durchgehend als

39. K. JANSEN-WINKELN, „Die Entwicklung der genealogischen Informationen nach dem Neuen Reich“, in M. Fitzenreiter (ed.), Genealogie - Realität und Fiktion von Identität (IBAES V), London, 2005, S. 137.

40. P. Munro, Die spätägyptischen Totenstelen (ÄF 25), Glückstadt, 1973, S. 174-175; D. A. Aston, „Dynasty 26, Dynasty 30 or Dynasty 27? In Search of the Funerary Archaeology of the Persian Period“, in A. Leahy - J. Tait (ed.), Studies in Ancient Egypt in Honour of H. S. Smith (OP 13), London, 1999, S. 17-22.

41. B. J. J. HaRING, „Libyans in the Late Twentieth Dynasty“, in R. J. Demarée-A. Egberts (ed.), Village Voices, Proceedings of the Symposium “Texts from Deir el Medina and their Interpretation”, Leiden, 1992, S. 71-80; ergänzend R. DemaréE, „Ramesside Administrative Papyri in the Civiche Raccolte Archeologiche e Numismatiche di Milano“, JEOL 42 (2010), S. 67-70; pl. III; Fr. CoLIN, Les Libyens en Égypte I, S. 90-96; K. JANSEN-WINKELN, „Ägyptische Geschichte im Zeitalter der Wanderungen von Seevölkern und Libyern", in E. Braun-Holzinger - H. Matthäus (ed.), Die nahöstlichen Kulturen und Griechenland an der Wende vom 2. zum 1. Jahrtausend v. Chr., Möhnesee, 2002, S. 135-140. 
Hinweise auf eine Bedrohung verstanden worden: die Arbeiter wagten nicht, ins Tal der Könige zu gehen ${ }^{42}$.

In jüngerer Zeit sind zwei Beiträge erschienen, nach denen es sich bei diesen „Besuchern“ nicht um (potentielle) Feinde gehandelt habe: F. Colin zufolge waren sie Händler, die die Nekropolenarbeiter mit Metall(geräten) belieferten, und manchmal zusätzliche Arbeitskräfte, jedenfalls keine Feinde ${ }^{43}$. Ähnlich sieht es S. Häggmann ${ }^{44}$ : Es handele sich um libysche Nomaden, die nach Theben kamen, um dort Handel zu treiben oder an religiösen Festen teilnehmen, und die Nekropolenarbeiter waren untätig, weil auch sie zu Markte wollten.

Diese Deutungen haben sehr wenig für sich ${ }^{45}$ :

a) Die übliche Formulierung $w z f$... $r$-h hıt nj Mšwš (u.ä.) zeigt schon, dass es sich nicht um normale Händler oder Marktbesucher handelt. Gegen Häggman ${ }^{46}$ bedeutet die Präposition $r$ - hst niemals ,because of“, sondern immer ,vor, an der Spitze, gegenüber, angesichts“. Dort, wo keine eigentlich lokale Bedeutung (,,befindlich vor, gegenüber") vorliegt, bezeichnet sie die Auswirkung der Gegenüberstellung, z.B. „standhalten vor, angesichts“ oder „fliehen vor". Wenn die Nekropolenarbeiter ,,angesichts“ der Libyer untätig sind, bedeutet das also ,aufgrund der Wirkung“, die von diesen Leuten ausgeht. Und die Parallele aus dem Jahr 1 Ramses' VI., wo es heißt wzf $r$ - h $3 t$ ps hrwy ,Untätigkeit angesichts des Feindes ${ }^{447}$ sollte eigentlich jeden Zweifel beseitigen, welche Bedeutung wzf $\ldots r$-h hst hat.

b) Kämen die Libyer tatsächlich nur als Händler, Marktbesucher oder Hilfskräfte, wäre nicht einzusehen, warum dann ,angesichts“ ihres Erscheinens die Arbeit ausfiel.

c) Sie werden konsequent als ethnische Gruppen bezeichnet.

d) Beim Jahreswechsel 10/11 unter Ramses IX. ${ }^{48}$ dauert die „Untätigkeit“ viele Tage. Hätte man nur auf eine Lieferung durch sie gewartet ${ }^{49}$, wäre das gänzlich unnötig. Und da sie sich offenbar auch über die Epagomenentage erstreckte, die vermutlich ohnehin arbeitsfrei waren ${ }^{50}$, ist klar, dass mit wzf nicht nur eine normale Arbeitsunterbrechung gemeint ist.

e) Es ist a priori sehr unwahrscheinlich, dass Teile der Stämme der Meschwesch und Rebu aus dem weit entferntem Nordwesten in Theben, tief in Oberägypten, als Händler oder Arbeiter auftreten. Sie sind auch während der gesamten übrigen Ramessidenzeit in Theben und in Deir el-Medina

42. S.u., Anm. 57.

43. Fr. CoLin, Les Libyens en Égypte I, S. 90-96.

44. S. Hägman, Directing Deir el-Medina. The External Administration of the Necropolis (USE 4), Uppsala, 2002, S. 293-308.

45. Die Belege aus der Whm-mswt-Zeit (ab Jahr 19 Ramses' XI.) können natürlich nicht als Hinweis auf friedlichen Umgang mit Libyern in Theben herangezogen werden, denn in dieser

Zeit beginnt entsprechend dem oben dargelegten die Herrschaft der Libyer über Ägypten.

46. S. HägGman, Directing Deir el-Medina, S. 297-298, n. 1988.

47. KRI VI, S. 342

48. KRI VI, S. 637-638.

49. Fr. Colin, Les Libyens en Égypte I, S. 92.

50. Vgl. W. HelcK, „Feiertage und Arbeitstage in der Ramessidenzeit“, JESHO 7 (1964), S. 159. 
niemals in dieser Funktion (oder in irgendeiner anderen) erschienen, und das trotz der verhältnismäßig sehr guten Beleglage für diese Siedlung.

f) Bis zu Ramses III. sind Meschwesch und Rebu expressis verbis als Feinde bezeugt, nach Ramses XI. sind sie die Herrscher Ägyptens: es wäre mehr als merkwürdig, sie in der Zwischenzeit als einfache Händler und Arbeiter in Theben anzutreffen. Die naheliegende Annahme ist vielmehr, dass es sich dabei um Vorgänge im Rahmen ihrer Durchdringung und Eroberung Ägyptens handelt.

Eine ,friedliche“ Interpretation der Anwesenheit von Libyern im Theben der späten 20. Dynastie ${ }^{51}$ ist unhaltbar. Dagegen ist es sehr naheliegend, Vorfälle aus dem Jahr 28 Ramses' III..$^{52}$ und dem Jahr 1 Ramses' VI. ${ }^{53}$, wo „Feinde“"erwähnt werden, die im letzteren Fall sogar einen ganzen Ort zerstört und die Einwohner verbrannt hatten, mit Kitchen ${ }^{54}$ als Aktionen ,äußerer“ Feinde $^{55}$ zu verstehen. Dafür spricht auch die Chronologie der Ereignisse: auf die ,äußeren“ Störungen folgen oft ,innere“ Unregelmäßigkeiten ${ }^{56}$, die offenbar dadurch bedingt (oder begünstigt) waren.

Die Frage ist allerdings, wer genau diese Eindringlinge waren. Die allgemeine Ansicht ist wohl die, dass es sich um „Wüstenbewohner" handelte, Beduinen und Libyer, die in den Oasen und an den Wüstenrändern siedelten, (potentiell) feindlich zwar, aber eher von marginaler Bedeutung ${ }^{57}$. Dagegen sprechen aber:

1. Die lange Dauer der „Störfälle“, die sich unter Ramses IX. über viele Jahre erstrecken und offenbar von der Staatsmacht nicht mehr kontrolliert werden können; dafür können kaum kleinere Gruppen verantwortlich sein.

51. Die aber in Einklang steht mit der heute üblichen erwähnten Tendenz in Archäologie und Geschichtswissenschaften, Kriege, Invasionen und generell ,äußere“ Störungen und Einflüsse kleinzureden oder wegzuerklären, s. dazu K. JANSEN-WINKELN, „Der Untergang des Alten Reiches“, Or 79 (2010), S. 299-301 (§ 17).

52. KRI V, S. 520,16-521,1.

53. KRI VI, S. 342-343.

54. K. A. Kitchen, „Les suites des guerres libyennes de Ramsès III“, RdE 36 (1985), S. 177179; ID., ,The Arrival of the Libyans in Late New Kingdom Egypt“, in A. Leahy (ed.), Libya and Egypt c1300-750 BC, London, 1990, S. 22.

55. Im ehesten Libyer, es könnten aber in dieser Zeit durchaus Verbände der "Seevölker“ mitgemacht haben.

56. Im Jahr 28 Ramses' III. sind „Feinde“ in Theben, im Jahr 29 beginnen die „Streiks“ aufgrund schlechter Versorgung; in den Jahren 8-15 sind feindliche Libyer in Theben, in den Jahren 16-18 gibt es die Grabräuberprozesse, im Jahr 3. Ramses X. sind wieder Libyer in Theben, wenige Monate danach findet wieder ein „Streik“ statt, u.ä., s. K. JANSEN-WINKELN, „Ägyptische Geschichte im Zeitalter der Wanderungen von Seevölkern und Libyern“, S. 139.

57. Vgl. etwa J. ČERNÝ, „Egypt: From the Death of Ramesses III to the End of the Twenty-First Dynasty“, in $C A H^{3}$ II/2, Cambridge, 1975, S. 616-619; K. A. KITCHEN, The Third Intermediate Period in Egypt, S. 247 (,the environs of Thebes itself were haunted by roaming Libyans and desert tribesfolk“); P. GRANDET, „Twentieth Dynasty“, in D. B. Redford (ed.), The Oxford Encyclopedia of Ancient Egypt II, Oxford, 2001, S. 541 (,,repeated Libyan raids“); J. vaN DIJK, „The Amarna Period and the Later New Kingdom", in I. Shaw (ed.), The Oxford History of Ancient Egypt, Oxford, 2000, S. 308 (,a group of Libyans, who continued to be a nuisance in the area“; „Libyan nomads disturbing the peace in Thebes“; „Libyan gangs“). J. OsıNG, in LÄ III, 1023, s.v. „Libyen, Libyer“, ( „ ... unklar ..., ob es sich um land- oder beutesuchende autochthone L[ibyer] oder um marodierende libysche Söldner des äg[yptischen] Heeres handelt"). 
2. In der gesamten ägyptischen Geschichte, vor und nach der 20. Dynastie, hat nie Gefahr für Oberägypten durch Angriffe aus den Oasen oder gar der Westwüste gedroht ${ }^{58}$. Die Tatsache, dass man Leute aus Ägypten in die Oasen verbannte, zeigt ja, wie leicht die Zugänge dorthin (und dorther) zu kontrollieren waren. Es ist viel wahrscheinlicher, dass es sich bei den Libyern und „Barbaren“ (hjstjw) ${ }^{59}$, die in Theben für Unruhe sorgten, um größere Truppenverbände handelte, die ins Niltal eingedrungen waren bzw. sich dort schon länger als Söldner befanden.

Wir hätten demnach folgende Situation: In Unterägypten gibt es in der späteren 20. Dynastie kaum noch Zeugnisse für Aktivitäten des Königs oder höherer Funktionäre: nach Ramses III. werden die unterägyptischen Quellen außerordentlich spärlich; die wenigen, die es noch gibt, stammen hauptsächlich aus Memphis und Heliopolis ${ }^{60}$. Und in Oberägypten, in Theben, weit im Landesinneren, finden wir Libyer als Feinde und Bedrohung. Ab der 21. Dynastie sind sie dann die Herren des Landes. Die mit Abstand wahrscheinlichste Erklärung ist also, dass die Libyer das Land erobert haben. Wie man sich das im einzelnen vorzustellen hat, bleibt offen. Naheliegend wäre ein Szenario, wie man es gegen Ende des Weströmischen Reiches vorfindet, wo barbarische Stämme und Truppenteile teils gewaltsam eindringen, teils als Verbündete und Söldner schon anwesend sind, sich dann aber zunehmend selbständig machen.

6. Die Libyerherrschaft hat also unmittelbar nach dem Neuen Reich begonnen. Sie wird in Oberägypten durch die Nubier unter Kaschta und Pianchi beendet, aber die Herrschaft der 25. Dynastie, seit Schabako auch in Unterägypten anerkannt, bedeutet keineswegs, dass die libyschen Lokalherrscher auch in Unterägypten entmachtet worden wären. Das Ägypten der 25. Dynastie ist kein zentralisierter Staat, sondern besteht aus Nubien und mehreren ägyptischen Teilgebieten, die unter der Oberherrschaft des nubischen Königs von eigenen Fürsten oder Königen regiert werden. Es gibt auch in dieser Zeit (libysche) Lokalfürsten und Kleinkönige ${ }^{61}$ in vielen unterägyptischen Städten ${ }^{62}$. In den Inschriften der assyrischen Könige Asarhaddon und Assurbanipal werden ausführliche Listen der ägyptischen Lokalfürsten (von den assyrischen Schreibern generell als šarru „König“ bezeichnet) gegeben $^{63}$, und auch in der "Traumstele“ des Tanutamun erscheinen die unterägyptischen Fürsten als wrw $n T \zeta-m h w$,Große des Nordlandes"64. Die

58. Das Bild des demographischen Drucks, das K. A. KITCHEN, „The Arrival of the Libyans“, S. 21-22 zeichnet (,penetration under demographic pressures“), ist ersichtlich eine moderne Vorstellung.

59. Das ist an dieser Stelle sicher die treffendste Wiedergabe dieses Wortes (s. Wb. III, S. 236, 1), nicht ,Wüstenbewohner“.

60. Vgl. KRI VI, passim.

61. K. JANSEN-WinKeln, Inschriften der Spätzeit, Teil III: Die 25. Dynastie, Wiesbaden, 2009,

S. $252-257$.

62. J. Yoyoтte, „Les principautés du Delta“; F. GomaA, Die libyschen Fürstentümer des Deltas.

63. H. U. ONASCH, Die assyrischen Eroberungen Ägyptens I (̈̈AT 27/1), Wiesbaden, 1994, S. 36.

64. Urk. III, S. 69,5; N.-C. Grimal, Quatre stèles napatéennes au Musée du Caire, JE 4886348866 (MIFAO 106), Kairo, 1981, S. 13,8; F. BREYER, Tanutamani. Die Traumstele und ihr Umfeld (ÄAT 57), Wiesbaden, 2003, S. 485, 10; 150-152. 
libyschen Lokalherrschaften sind weder von Nubiern noch von Assyrern beseitigt worden, sicher mit gutem Grund: Ein Land, das derart in einzelne Territorien zerfallen ist, läßt sich leichter von außen beherrschen.

Diese Situation ändert sich erst in der 26. Dynastie: Psametik I., selbst zweifellos libyscher Herkunft, beseitigt die Lokalfürstentümer: die Titel der Lokalfürsten verschwinden und die entsprechenden Städte und Gebiete werden wieder von einem $h^{\prime}{ }^{\prime} t j{ }^{-}{ }^{\prime}$ geleitet. Es ist auch deutlich, dass unter dem neuen Königtum Leute ganz unterschiedlicher Herkunft, Ägypter, Libyer und Fremde, bis in höchste (militärische und zivile) Ränge aufsteigen können. Im übrigen bleiben auch in der 26. Dynastie bestimmte Strukturen der Libyerzeit bis zum Ende der 26. Dynastie erhalten, z.B. der oberägyptische Teilstaat der Gottesgemahlin, eine unmittelbare Fortsetzung des Gottesstaates der 3. Zwischenzeit. Auch die Libyer selbst siedeln natürlich nach wie vor im Delta ${ }^{65}$.

7. Die durch die Herrschaft der Libyer neugebildeten Strukturen waren offenbar recht stabil, vieles davon hat Jahrhunderte Bestand gehabt. Aber welche Position hatten Ägypter und Libyer jeweils darin? Es ist sehr wahrscheinlich, dass die Libyer sich vorwiegend im Delta angesiedelt hatten ${ }^{66}: \mathrm{Ob}$ sie nun als Eroberer oder als friedliche Einwanderer gekommen sind, das Delta war zunächst ihre unmittelbare Nachbarschaft und zudem für viehzüchtende halbnomadische Stämme besonders geeignet. Auch finden sich libysche Lokalherrschaften mit einem Stammesfürsten ( $w r$ ( ) an der Spitze ausschließlich in Unterägypten. Das muß keineswegs bedeuten, dass es eine strikte Trennung der Siedlungsgebiete gab; man wird aber annehmen dürfen, dass die Libyer eher in Unterägypten in größeren Gruppen ansässig und dort der dominierende Bevölkerungsteil waren.

Eine gewisse Bestätigung dafür kann man darin sehen, dass aus Unterägypten von der 21 . bis zur 25 . Dynastie außerordentlich wenige Privatdenkmäler überliefert sind (nur Serapeumstelen und Schenkungsstelen sind reichlicher vorhanden $)^{67}$ : Offenbar legte die libysche Oberklasse wenig Wert auf diese Art der ägyptischen Tradition. Die Aufgaben und Tätigkeiten der Libyer lagen vermutlich vorwiegend im militärischen Bereich, sie waren Krieger. Das ist zwar weitgehend unstrittig ${ }^{68}$, es ist allerdings nur zu erschließen, wir haben darüber keinerlei zeitgenössische Aussagen in den Quellen. Man kann ihnen allenfalls entnehmen, dass die libyschen Häuptlinge bzw. „Fürsten“ auch Militärführer waren. Für alle weitergehende Vermutungen ist die nächste Quelle Herodot: Bei ihm findet sich die berühmte Darstellung

65. Und es ist gut möglich, dass es auch noch lokale libysche Machthaber gab: bei den Deltadynasten der 27.-30. Dynastie, die maßgeblich an den Aufständen gegen die Perserherrschaft beteiligt waren, handelte es sich vermutlich um Libyer, vgl. Fr. CoLIN, Les Libyens en Égypte I, S. 133-134, und auch in den Oasen halten sich noch lange libysche Fürsten, ibid., S. 141-161.

66. Vgl. auch A. Leahy, „The Libyan Period in Egypt“, S. 56: „The picture of population distribution that emerges from the very patchy evidence is of a Libyan Delta and a largely Egyptian South.“

67. Und angesichts der Fülle von Privatdenkmälern, die aus dem Unterägypten der 26. Dynastie überliefert sind, kann das kein Zufall sein, der nur durch die unterschiedlichen Naturräume bedingt ist.

68. S. dazu schon E. MEYER, Gottesstaat, Militärherrschaft und Ständewesen in Ägypten (SPAW), 1928, S. 513-529. 
(II.164), dass es bei den Ägyptern 7 erbliche Berufe (Genea) gegeben habe: Priester, Krieger, Rinderhirten, Schweinehirten, Händler, Dolmetscher und Schiffer. Die Krieger (Machimoi) teilt er in Kalasirier (vorwiegend im östl. Delta wohnend) und Hermotybier (im westl. Delta): sie sind nur Krieger und dürfen kein Handwerk lernen. Diese Machimoi werden generell, und sicher zurecht, auf die libysche Kriegerklasse der 3 . Zwischenzeit zurückgeführt: auch dort treffen wir zwei Hauptstämme an, die vorwiegend im Delta siedeln. Es wäre a priori auch nicht unwahrscheinlich, dass eine zahlenmäßig sicher sehr viel kleinere Ethnie, die ein größeres Land beherrscht, sich das Recht auf Waffen exklusiv sichert. Herodot sagt weiter (II.168), dass jeder der Machimoi 12 Aruren Land hatte, von deren Einkünften sie lebten; die eigentlichen Produzenten waren dann Ägypter.

Einen Einblick in die Mentalität dieser libyschen Krieger kann vielleicht die spätere „Heldenepik“ vermitteln, die aus ptolemäischer und römischer Zeit überliefert ist: die demotischen Erzählungen um Petubastis und Inaros, die nach Namen und historischen Umständen auf die spätere 3. Zwischenzeit verweisen. Diese Geschichten haben mit der älteren ägyptischen Tradition nichts gemein, und als Ursprung wird man sicher eher mündliche Erzählungen der Libyer vermuten als homerischen Einflu $\beta^{69}$.

Die in unseren Quellen greifbaren Ägypter (der Oberschicht) sind dagegen in aller Regel Priester und daneben oft auch Schreiber bzw. in Funktionen, die Schriftlichkeit erfordern. Die Aufgaben der ägyptischen Oberschicht hätten damit im Bereich von Religion, Kult, Schrift und kulturellen Tätigkeiten allgemein gelegen. Diese Priesterämter sind überwiegend erblich, aufgrund ausführlicher Filiationsangaben sind ganze Priesterdynastien bekannt. Diese Verhältnisse, einmal entstanden, hatten auch für die ägyptische Oberschicht zweifellos ihre Vorteile und werden ihr erhaltenswert erschienen sein. Tatsächlich ändert sich das ägyptische „Kastenwesen“, das vermutlich unter dem Einfluß der Libyerherrschaft entstanden oder zumindest stark ausgebaut worden ist, in der gesamten Spätzeit nicht mehr grundsätzlich.

Insgesamt scheint sich ein System entwickelt zu haben, das dem Feudalismus des Mittelalters nicht ganz unähnlich ist: eine Kriegerklasse, die von der Arbeit anderer lebt und Priester, die zugleich die Träger von Bildung und Schriftkultur sind.

Das eingangs erwähnte Paradoxon, dass die Libyer in der Libyerzeit weitgehend unsichtbar sind, läßt sich daher vielleicht durch die Besonderheiten dieser neuen Gesellschaftsordnung erklären: Unsere Quellen stammen ganz überwiegend aus dem sakralen (Tempel) und funerären Bereich, und dafür waren die (ägyptischen) Priester zuständig. Und die libyschen Krieger lebten ihrerseits von der Produktion anderer und hinterließen somit keine archäologischen Spuren.

8. Die durch die Libyerzeit geprägte gesellschaftliche Entwicklung ist von noch längerer Dauer gewesen als die politische, nicht zuletzt durch diese Herausbildung eines Berufspriestertums. Priesterschaft und Tempel sind eigentlich erst seit dieser Zeit Institutionen außerhalb oder neben den

69. Vgl. dazu auch J.Fr. QuACK, Einführung in die altägyptische Literaturgeschichte III, Die demotische und gräko-ägyptische Literatur (EQAE 3), Münster, 2005, S. 60. 
anderen staatlichen Einrichtungen, und dies ist dann eine der Hauptursachen für die Auseinanderentwicklung der Sphären von Diesseits und Jenseits in der ägyptischen Kultur, die für das gesamte 1. Jahrtausend so charakteristisch ist $^{70}$. Auch die Trennung der Landesteile hatte weitreichende Folgen, wie sich schon an der Auseinanderentwicklung der Kursivschriften in Oberund Unterägypten zeigt. Und die Libyerzeit steht letztlich auch am Beginn der Marginalisierung Oberägyptens, eine Tendenz, die dann in der späteren 26. Dynastie überaus deutlich wird und bis zum Ende der pharaonischen Kultur andauert.

Entgegen dem ersten Anschein ist es also durchaus so, dass die Herrschaft der Libyer das Ägypten des 1 . Jahrtausends nachhaltig geprägt hat.

70. S. K. JANSEN-WINKELN, „Bild und Charakter der ägyptischen 26. Dynastie“, AltorForsch 28 (2001), S. 171-174. 